\title{
Chronic Physical Illness Affects Emotion Regulation Process: A Case of HIV/AIDS
}

\author{
Tiwari, G. $\mathrm{K}^{1 *}$
}

\section{ABSTRACT}

The chronic physical and mental clinical conditions are characterized by some or other sorts of emotional problems. People suffering from these diseases find themselves incapable to regulate their emotions resulting from pain, hopelessness, lack of care etc. The individuals suffering from Human Immunodeficiency Virus/Acquired Immune deficiency Syndrome (HIV/AIDS) face many types of physical and mental health problems. The central goal of the present study was to compare and contrast the characteristic features of emotion regulation processes of the participants and uncover the intricacies of these processes rampant in the people living with chronic disease of HIV/AIDS. The two strategies of emotion regulation were chosen for this purpose i.e., emotional suppression and emotional appraisal. Eighty participants took part in the study in which forty were diagnosed male and female patients with HIV/AIDS and the rest forty were normal adults with no known/reported chronic disease of any kind. The results of the study evinced that the clinical and non-clinical participants significantly differed in their mean scores on emotional suppression and cognitive reappraisal strategies of emotion regulation. The results have been discussed in the light of current theories of emotion regulation along with their implications for future research.

Keywords: Emotion Regulation, Emotional Suppression, Cognitive Reappraisal, HIV/AIDS, Chronic Disease.

According to World Health organization, the number of people living with HIV/AIDS was about 35.0 million by the end of 2013 as compared to 29.8 million in 2001 (Global Observatory Data, 2015). This reflects continued transmission of the disease despite reductions in incidence, and the benefits of expanded access to antiretroviral, which have helped to reduce the number of people dying from AIDS. Thus, HIV/AIDS remains to occupy the position of an epidemic even today. In addition to many physical symptoms, HIV/AIDS patients also face psychological illhealth challenges. The diagnosis of HIV/AIDS for the first time ensues to be like a trauma for the persons and acts as a precursor to develop and maintain various types of reactions to stress and trauma, such as fear, fury, denial, depression, withdrawal, panic, extreme avoidance behaviors, impairment of ability to love and work. Moreover, such people express emotional distress and

\footnotetext{
${ }^{1}$ Assistant Professor, Department of Psychology, School of Humanities \& Social Sciences, Doctor Harisingh Gour Vishwavidyalaya, Sagar *Corresponding Author

(c) 2015 I G Tiwari; licensee IJIP. This is an Open Access Research distributed under the terms of the Creative Commons Attribution License (http://creativecommons.org/licenses/by/2.0), which permits unrestricted use, distribution, and reproduction in any Medium, provided the original work is properly cited.
} 


\section{Chronic Physical Illness Affects Emotion Regulation Process: A Case of HIV/AIDS}

dysfunctional attitudes toward illness, depressive symptoms, anxiety and psychosomatic disorder constituting the most common psychiatric problems of HIV/AIDS patients. All these psychiatric problems incorporate some sort of emotional irregularities which ultimately either originate a new health challenge or maintain and consolidate the existing one.

Emotion regulation entails increasing, maintaining, or decreasing positive and negative emotions and often involves changes in emotional responding. The emotional changes that are produced by emotion regulation may or may not bring people closer to the emotional state that they desire. Even, some forms of emotion regulation incongruously bring about the outcomes that people wish to avoid (Wegner, Erber, \& Zanakos, 1993). In either case, a coordinated set of behavioral, experiential, and physiological responses to attend the emotions ensues. Emotion regulation refers to the processes by which we influence which emotions we have, when we have them, and how we experience and express those (Gross, 1998). Because emotions are multi-componential processes that unfold over time, emotion regulation involves changes in "emotion dynamics", or the latency, rise time, magnitude, duration, and offset of responses in behavioral, experiential, or physiological domains. Emotion regulation also involves changes in how response components are interrelated as the emotion unfolds, such as when increases in physiological responding occur in the absence of overt behavior. Emotion regulation strategies vary in nature and consequences and impacts people's emotions may be recruited in the service of emotion regulation (Thompson, 1990, Tiwari, 2013).

It has been reported that the ability to effectively regulate undesired affective states are affected in many type of mental disorders (DSM-5; APA, 2013). The individuals suffering from depression frequently report troubles in identifying their emotions (Rude \& McCarthy, 2003), compassionately supporting themselves when suffering from negative emotions (Hofmann, Grossman, \& Hinton, 2011), and effectively modifying their emotions (Kassel, Bornovalova \& Mehta, 2007). Moreover, it has been shown that symptoms of depression are positively associated with ruminating/brooding, catastrophizing, and expressional suppression (Aldao, Nolen-Hoeksema, \& Schweizer, 2010), which have been conceptualized as ineffective attempts to avoid negative emotions (Berking \& Wupperman, 2012). Thus, emotion regulation strategies of an individual significantly affect the way positive and negative emotions are understood, managed and expressed in both normal and psychopathological conditions. The negative emotionality ensuing depression and other emotional disorders are maintained, enhanced or sometimes magnified by these processes.

The researchers have found that a person's positive belief in their ability to successfully modify their own negative affect can predict future reductions in depression (Kassel et al., 2007), and the use of regulation strategies generally considered to be maladaptive can predict depressive symptoms (Aldao \& Nolen-Hoeksema, 2012). Numerous studies have come to the conclusion that emotion regulation skill deficits contribute to the development and maintenance of anxiety disorders (Campbell-Sills, Ellard, \& Barlow, 2014). For example, in a nonclinical sample of 631 


\section{Chronic Physical Illness Affects Emotion Regulation Process: A Case of HIV/AIDS}

participants, emotion regulation skill deficits significantly predicted subsequent anxiety symptoms severity during a 2-week interval (Berking, Orth, Wupperman, Meier, \& Caspar, 2008). Research involving clinical samples has found that individuals suffering from generalized anxiety disorder (GAD) display deficits in emotional clarity, a poorer understanding of emotions, greater negative reactivity to emotions, as well as less acceptance and less successful management of emotions (McLaughlin, Mennin, \& Farach, 2007; Mennin, Heimberg, Turk, \& Fresco, 2005). Research on clinical samples also indicated that individuals meeting criteria for panic disorder report difficulties identifying, labeling, accepting, and tolerating undesired emotions (Baker, Holloway, Thomas, Thomas, \& Owens, 2004 ; Naragon- Gainey, 2010).

The individuals diagnosed with social anxiety disorder have been reported to show more difficulty describing and identifying emotions as compared to healthy controls (Turk, Heimberg, Luterek, Mennin, \& Fresco, 2005), experience higher levels of shame (Fergus, Valentiner, McGrath, \& Jencius, 2010), and display greater difficulty accepting emotional experiences when they are confronted with negative events (Kashdan \& Steger, 2006). In the same way, the individuals suffering from specific phobic symptoms exhibit increased use of avoidance, selfaccusation, rumination, catastrophizing, and low reliance on positive reappraisals (Kraaij, Garnefski, \& Van Gerwen, 2003). Likewise, the symptom severity and impairment of posttraumatic stress disorder persons have been found to be associated with a lack of emotional clarity, a lack of emotional acceptance, and difficulties engaging in goal-directed behavior and effective emotion regulation strategies (Ehring \& Quack, 2010; Tull, Barrett, McMillan, \& Roemer, 2007). Emotion regulation difficulties have also been shown to be significantly associated with symptoms of substance abuse (Staiger, Melville, Hides, Kambouropoulos, \& Lubman, 2009), eating disorders (Svaldi, Griepenstroh, Tuschen-Caffi er, \& Ehring, 2012), emotional disorders (Ellard, Fairholme, Boisseau, Farchione, \& Barlow, 2010), borderline personality disorders (Hasin, Stinson, Ogburn, \& Grant, 2007), somatoform disorders (Sifneos, 1973), childhood psychopathology (Walcott \& Landau, 2004) and a variety of internalizing (social withdrawal, depression, and anxiety) and externalizing behaviors (aggression, anger, and behavior problems) (Kim \& Cicchetti, 2010). In summary, there is strong empirical evidence that emotion regulation deficits are associated with mental disorders and that emotion regulation deficits contribute significantly to the development and maintenance of these disorders.

In this backdrop of the findings associated with the role of emotion regulation in the genesis, maintenance and expression of mental disorders, the present study attempted to understand the prevalence and use of emotional suppression and cognitive reappraisal strategies of emotion regulation in the people suffering from HIV/AIDS. The basic research question of the study is to ascertain whether these people differ in their use of the two emotion regulation processes. 


\section{Chronic Physical Illness Affects Emotion Regulation Process: A Case of HIV/AIDS}

\section{Hypotheses}

The following hypotheses have been postulated for the present study:

1. The participants suffering from HIV/AIDS will demonstrate higher mean score of emotional suppression as compared to the normal healthy participants.

2. The normal healthy participants will evoke higher mean score on cognitive reappraisal measure as compared to their clinical counterparts.

\section{METHODS AND PROCEDURE}

\section{Sample}

Forty diagnosed HIV/AIDS patients registered in Antiretroviral Therapy (ART) Center Bundelkhand Medical College, Sagar, M. P. comprising twenty seven males $(\mathrm{M}=35.63$, SD = 4.58) and thirteen females $(\mathrm{M}=33.69, \mathrm{SD}=3.57)$ and forty postgraduate students from Doctor Harisingh Gour University Sagar, Madhya Pradesh, India comprising twenty eight male $(\mathrm{M}=$ 28.00 , $\mathrm{SD}=4.78)$ and twelve females $(\mathrm{M}=24.17$, $\mathrm{SD}=5.99)$ served as the participants in the study. Age range of the participants was from 19-40 years. They belonged to different socioeconomic backgrounds predominantly lower middle class.

\section{Psychometric Tool}

Hindi version (translated by Khetrapal, Gupta \& Baijal, 2007) of Emotion Regulation Questionnaire (ERQ) developed and standardized by Gross \& John (2003) designed to assess individual differences in the habitual use of two emotion regulation strategies: cognitive reappraisal and expressive suppression was used to assess the emotion regulation of the participants. It has acceptable levels of psychometric properties like reliability and validity along with a history of wide applications in research around the globe. It consists of 10 items of which six belonged to cognitive reappraisal and the rest four to expressive suppression with seven point Likert scale ranging from strongly disagree to the strongly agree from extreme left to extreme right, respectively. Higher scores indicated higher levels of cognitive reappraisal and expressive suppression of the participants.

\section{Procedure}

Before the administration of the questionnaire, overall planning was done. Written permissions were requested from ART Centre to conduct the study on HIV/AIDS patients as per the ethical standards expected to be observed for the study of humans. Written informed consent from the participants was sought. The participation in the study was voluntary and the participants were fully aware of the fact that they could withdraw their participation in the study at any point of time. The study started with the establishing rapport with the participants followed by imparting of the instructions and administration of the Emotion Regulation Questionnaire on the participants. The participants had to write the appropriate number on the right margin of each statement which represented his/her most closest feeling/thought about the meaning/content inherent in each statement of the scale. The collected data were compiled and mean, standard 


\section{Chronic Physical Illness Affects Emotion Regulation Process: A Case of HIV/AIDS}

deviation (SD) and t-test were computed for the sake of comparison and discussion of the results and the testing of hypotheses.

\section{RESULTS}

Results of the study indicated that HIV/AIDS patients acquired higher mean emotional suppression score $(\mathrm{M}=23.75, \mathrm{SD}=1.99)$ as compared to the normal participants $(\mathrm{M}=10.48$, $\mathrm{SD}=1.74)$ whereas they showed lower mean scores $(\mathrm{M}=9.35$, $\mathrm{SD}=2.13)$ for cognitive reappraisal in comparison to their normal counterparts $(\mathrm{M}=26.35, \mathrm{SD}=2.08)$. Thus, the clinical group significantly differed in these measures of emotional regulation as compared to normal healthy participants.

Table 1: Mean emotional suppression and cognitive reappraisal scores of HIV/AIDS and normal participants

\begin{tabular}{lllll} 
S. No. & Participants & Emotional Regulation & Mean & SD \\
\hline \multirow{2}{*}{ 1. } & \multirow{2}{*}{ AIDS Patients } & Emotional suppression & 23.75 & 1.99 \\
& & Cognitive Reappraisal & 9.35 & 2.13 \\
2. & \multirow{2}{*}{ Normal } & Emotional suppression & 10.48 & 1.74 \\
& & Cognitive Reappraisal & 26.35 & 2.08 \\
\hline
\end{tabular}

Mean scores of emotional suppression and cognitive reappraisal of the participants suffering from HIV/AIDS were compared with the mean scores of the normal participants through t-test. The analyses demonstrated that the mean scores of emotional suppression of the participants with HIV/AIDS and normal differed significantly $(\mathrm{t}=35.35 \mathrm{df}=39, p=.001)$. In the case of the mean scores of cognitive reappraisal, the two groups of the participants also showed statistically significant difference $(\mathrm{t}=38.01, \mathrm{df}=39, p=.001)$. These results have been depicted lucidly in Table 1 and Figure 1.






\section{Chronic Physical Illness Affects Emotion Regulation Process: A Case of HIV/AIDS}

\section{DISCUSSION}

The results of the present study establish the fact that chronic disease like HIV/AIDS shaped the nature and extent of the emotion regulation processes. It was demonstrated with the results and findings of the study that psychological consequences of being inflicted with HIV/AIDS regulated the basic nature of emotional suppression and cognitive reappraisal of the participants. This is self-evident that the persons who have been suffering from such chronic disease would unavoidably develop many psychological problems like depression, anxiety, emotional dysfunctioning, to mention a few. These symptoms are associated and maintained by these irregularities in emotion regulation processes. Results of the study indicated that HIV/AIDS patients emitted higher mean emotional suppression score as compared to their normal participants whereas they showed lower mean scores for cognitive reappraisal in comparison to their normal counterparts. Mean scores of emotional suppression and cognitive reappraisal of the participants suffering from HIV/AIDS were compared with the mean scores of the normal participants through t-test. The analyses demonstrated that the mean scores of emotional suppression of the participants with HIV/AIDS and normal differed significantly. In the case of the mean scores of cognitive reappraisal, the two groups of the participants also showed statistically significant difference.

The excess use of emotional suppression is a reliable indicator of poor mental health and psychological functioning. The irregularities in emotional suppression also acts as a precursor for developing many types of mental disorders like depression, anxiety disorders, low selfforgiveness (Mudgal \& Tiwari, in press), lowered body image satisfaction and life satisfaction (Jain \& Tiwari, in press) and a variety of emotional disturbances. Contrarily, cognitive reappraisal leads to healthy adjustment and positive psychological functioning (Hofmann, Grossman, \& Hinton, 2011; Kassel, Bornovalova \& Mehta, 2007; McLaughlin, Mennin, \& Farach, 2007). The results were in consonance of the expected mental health outcomes of the persons facing the chronic disease like HIV/AIDS. Such results have been reported in many studies (Aldao \& Nolen-Hoeksema, 2012; Aldao, Nolen-Hoeksema, \& Schweizer, 2010; Berking \& Wupperman, 2012; Berking, Orth, Wupperman, Meier, \& Caspar, 2008; Campbell-Sills, Ellard, \& Barlow, 2014; Rude \& McCarthy, 2003).

\section{CONCLUSIONS AND SUGGESTIONS FOR FUTURE RESEARCH}

On the basis of above discussion of the results, it can be concluded that the HIV/AIDS patients demonstrated dysregulation of their emotions and emotional functioning as compared to normal healthy participants. The clinical participants showed higher mean scores on emotional suppression as compared to their normal counterparts, whereas for cognitive reappraisal, they exhibited lower mean score. Excess use of emotional suppression is a reliable indicator of poor mental health and psychological functioning along with precursor of depression, anxiety disorders and a variety of emotional disturbances as opposed to cognitive reappraisal which leads to healthy adjustment and positive psychological functioning. The results were in consonance of the expected mental health outcomes of the persons facing the chronic disease like HIV/AIDS. It 


\section{Chronic Physical Illness Affects Emotion Regulation Process: A Case of HIV/AIDS}

is expected that emotion regulation-based therapeutic intervention techniques would do well in the case of people suffering from HIV/AIDS. There are some limitations of this study as it involved a very small sample and was limited on limited geographical area. The findings of the study were based on only one quantitative measure. The future researches may be conducted comprising relatively a large sample with diversified geographical and socio-cultural backgrounds and with multiple variables. Another suggestion for future research is that they should involve qualitative methods as the questionnaires did not provide them with a complete opportunity to express themselves. The results and conclusions of the study have important implications for future researchers, planners, policy makers and health professionals who are associated with the services of people inflicted with diseases like HIV/AIDS.

\section{Acknowledgement}

I acknowledge the cooperation and assistance of Dr. Abhay Tirkey, Nodal Officer, ART Center, Sagar, M. P., his colleague Sri Anupam Bohare, Counsellor and National AIDS Control Organization (NACO), India, in extending permissions to conduct the study. I also express my heartiest gratitude towards the contributions of Miss Sujata Mudgal and Mr. Pankaj Jain, both are research scholars at the Department of Psychology, School of Humanities \& Social Sciences, Doctor Harisingh Gour Vishwavidyalaya, Sagar, M. P., India, who helped in collection and compilation of the data.

\section{REFERENCES}

Aldao, A., \& Nolen-Hoeksema, S. (2012). When are adaptive strategies most predictive of psychopathology? Journal of Abnormal Psychology, 121 (1), 276-281.

Aldao, A., Nolen-Hoeksema, S., \& Schweizer, S. (2010). Emotion-regulation strategies across psychopathology: A meta-analytic review. Clinical Psychology Review, 30 (2), 217-237.

American Psychiatric Association. (2013). Diagnostic and statistical manual of mental disorders (5th ed.). Washington, DC: Author.

Baker, R., Holloway, J., Thomas, P. W., Thomas, S., \& Owens, M. (2004). Emotional processing and panic. Behaviour Research and Therapy, 42 (11), 1271-1287.

Berking, M., Orth, U., Wupperman, P., Meier, L., \& Caspar, F. (2008). Prospective effects of emotion regulation on emotional adjustment. Journal of Counseling Psychology, 55 (4), 485-494.

Berking, M., \& Wupperman, P. (2012). Emotion regulation and mental health: Recent findings, current challenges, and future directions. Current Opinion in Psychiatry, 25 (2), 128-134.

Campbell-Sills, L., Ellard, K., \& Barlow, D. H. (2014). Emotion regulation in anxiety disorders. In J. J. Gross (Ed.), Handbook of emotion regulation (2nd ed., pp. 393-412). New York, NY: Guilford Press.

Ehring, T., \& Quack, D. (2010). Emotion regulation diffi culties in trauma survivors: The role of trauma type and PTSD symptom severity. Behavior Therapy, 41 (4), 587-598.

Ellard, K. K., Fairholme, C. P., Boisseau, C. L., Farchione, T. J., \& Barlow, D. H. (2010). Unified protocol for the transdiagnostic treatment of emotional disorders: Protocol 


\section{Chronic Physical Illness Affects Emotion Regulation Process: A Case of HIV/AIDS}

development and initial outcome data. Cognitive and Behavioral Practice, 17 (1), 88101.

Fergus, T. A., Valentiner, D. P., McGrath, P. B., \& Jencius, S. (2010). Shame-and guiltproneness: Relationships with anxiety disorder symptoms in a clinical sample. Journal of Anxiety Disorders, 24 (8), 811-815.

Gross, J. J. (1998). The emerging field of emotion regulation: An integrative review. Review of General Psychology, 2, 271-299.

Gross, J. J., \& John, O.P. (2003). Individual differences in two emotion regulation processes: Implications for affect, relationships, and wellbeing. Journal of Personality and Social Psychology, 85, 348-362.

Hasin, D. S., Stinson, F. S., Ogburn, E., \& Grant, B. F. (2007). Prevalence, correlates, disability, and comorbidity of DSM-IV alcohol abuse and dependence in the United States: Results from the national epidemiologic survey on alcohol and related conditions. Archives of General Psychiatry, 64 (7), 830-842.

Hofmann, S. G., Grossman, P., \& Hinton, D. E. (2011). Loving-kindness and compassion meditation: Potential for psychological interventions. Clinical Psychology Review, 31 (7), 1126-1132.

Jain, P. \& Tiwari, G. K. (In press). Body image satisfaction and life satisfaction in HIV/AIDS patients. The International Journal of Indian Psychology.

Kashdan, T. B., \& Steger, M. F. (2006). Expanding the topography of social anxiety: An experience sampling assessment of positive emotions, positive events, and emotion suppression. Psychological Science, 17 (2), 120-128.

Kassel, J. D., Bornovalova, M., \& Mehta, N. (2007). Generalized expectancies for negative mood regulation predict change in anxiety and depression among college students. Behaviour Research and Therapy, 45 (5), 939-950.

Kim, J., \& Cicchetti, D. (2010). Longitudinal pathways linking child maltreatment, emotion regulation, peer relations, and psychopathology. Journal of Child Psychology and Psychiatry,51 (6), 706-716.

Khetrapal, N., Gupta, R. \& Baijal, S. (2007). Hindi Version of Emotion regulation Questionnaire of Gross \& John, 2003, Centre for Behavioural \& Cognitive Sciences, University of Allahabad, India Retrieved from http://spl.stanford.edu/pdfs/ERQ/Hindi.pdf

Kraaij, V., Garnefski, N., \& Van Gerwen, L. (2003). Cognitive coping and anxiety symptoms among people who seek help for fear of flying. Aviation, Space, and Environmental Medicine,74 (3), 273-277.

McLaughlin, K. A., Mennin, D. S., \& Farach, F. J. (2007). The contributory role of worry in emotion generation and dysregulation in generalized anxiety disorder. Behaviour Research and Therapy, 45 (8), 1735-1752.

Mennin, D. S., Heimberg, R. G., Turk, C. L., \& Fresco, D. M. (2005). Preliminary evidence for an emotion regulation defi cit model of generalized anxiety disorder. Behaviour Research and Therapy, 43 (10), 1281-1310. 


\section{Chronic Physical Illness Affects Emotion Regulation Process: A Case of HIV/AIDS}

Mudgal, S. \& Tiwari, G. K. (In press). Self-forgiveness and life satisfaction in people living with HIV/AIDS. The International Journal of Indian Psychology.

Naragon-Gainey, K. (2010). Meta-analysis of the relations of anxiety sensitivity to the depressive and anxiety disorders. Psychological Bulletin, 136 (1), 128.

Rude, S. S., \& McCarthy, C. T. (2003). Emotional functioning in depressed and depression vulnerable college students. Cognition and Emotion, 17 (5), 799-806.

Sifneos, P. E. (1973). The prevalence of 'alexithymic' characteristics in psychosomatic patients. Psychotherapy and Psychosomatics, 22 (2- 6), 255-262.

Staiger, P. K., Melville, F., Hides, L., Kambouropoulos, N., \& Lubman, D. I. (2009). Can emotion focused coping help explain the link between posttraumatic stress disorder severity and triggers for substance use in young adults? Journal of Substance Abuse Treatment, 36 (2), 220-226.

Svaldi, J., Griepenstroh, J., Tuschen-Caffi er, B., \& Ehring, T. (2012). Emotion regulation deficits in eating disorders: A marker of eating pathology or general psychopathology? Psychiatry Research, 197 (1), 103-111.

Thompson, R. A. (1990). Emotion and self-regulation. In R. A. Thompson (Ed.), Socioemotional development. Nebraska Symposium on Motivation (Vol. 36, pp. 367-467). Lincoln: $\quad$ University of Nebraska Press.

Tull, M. T., Barrett, H. M., McMillan, E. S., \& Roemer, L. (2007). A preliminary investigation of the relationship between emotion regulation difficulties and posttraumatic stress symptoms. Behavior Therapy, 38 (3), 303-313.

Turk, C. L., Heimberg, R. G., Luterek, J. A., Mennin, D. S., \& Fresco, D. M. (2005). Emotion dysregulation in generalized anxiety disorder: A comparison with social anxiety disorder. Cognitive Therapy and Research, 29 (1), 89-106.

Tiwari, G. K. (2013). Emotional suppression and eyewitness memory. JIGYASA, 4 (4), 196-203.

Walcott, C. M., \& Landau, S. (2004). The relation between disinhibition and emotion regulation in boys with attention deficit hyperactivity disorder. Journal of Clinical Child and Adolescent Psychology, 33 (4), 772-782.

Wegner, D. M., Erber, R., \& Zanakos, S. (1993). Ironic processes in the mental control of mood and mood related thought. Journal of Personality and Social Psychology, 58, 409-418.

World Health Organization (2015). Global Health Observatory Data. Retrieved from http://www.who.int/gho/hiv/epidemic_status/cases_all_text/en/ 